\title{
Is Age an Independent Predictor of High-Grade Histopathology in Women Referred for Colposcopy after Abnormal Cervical Cytology?
}

\author{
Chalita Kingnate, Amornrat Supoken, Pilaiwan Kleebkaow, Bundit \\ Chumworathayi, Sanguanchoke Luanratanakorn, Chumnan Kietpeerakool*
}

\begin{abstract}
This study was conducted to determine whether advancing age is an independent predictor of increased risk of high-grade pathologies among women referred for colposcopy after abnormal cervical cytology. Medical records were reviewed for women with abnormal cervical cytology who underwent colposcopy at Khon Kaen University Hospital. Logistic regression was used to determine the independent impact of age on the risk of high-grade pathologies. Mean age of the women was 42.8 years. Of 482 women, $97(20.1 \%)$ were postmenopausal, and 92 (19.1\%) were nulliparous. The rate of high-grade pathologies included cervical intraepithelial neoplasia 2-3, 99 (20.5\%), adenocarcinoma in situ, $4(0.8 \%)$, cervical cancer, $30(6.2 \%)$, and endometrial cancer, $1(0.2 \%)$. The prevalence of significant lesions was $26.9 \%$ (95\% CI, $23.1 \%-31.2 \%)$. In total, 31 women had cancers $(6.4 \%$; $95 \%$ CI, 4.4\%-9.0\%). When controlling for smear types and parity, age was noted to be a significant independent predictor of high-grade histopathology. Women older than 35-40 years were approximately 2 times as likely to have severe histopathology as the younger women. This study illustrates the substantial risk of underlying significant lesions especially invasive cancer in Thai women with abnormal cervical cytology. Age was a significant independent factor predicting the risk of high-grade pathologies.
\end{abstract}

Keywords: Cervical cytology - cervical cancer - cervical intraepithelial neoplasia - risk factor

Asian Pac J Cancer Prev, 16 (16), 7231-7235

\section{Introduction}

Persistent infection, caused by high-risk human papillomavirus (HR-HPV), is the pivotal step in cervical carcinogenesis (Dalstein et al., 2003; Chen et al., 2011). Persistent HR-HPV infection is more common in older women (Bosch and Harper 2006; Kjaer et al., 2010; Kang et al., 2014). In the recent study by Kang et al. (2014), the risk of not clearing a HR-HPV infection at one-year among women younger than 30 years was $20 \%$, but in women $30-39$ years, approximately $50 \%$ of the HR-HPV infections persisted. Additionally, HR-HPV infection had spontaneously regressed in only one-third of women around 60 years. Immunological dysregulation is likely an important determinant of risk of HPV persistence among older women (Rodriguez et al., 2011). These findings therefore theoretically contribute to the increased likelihood of developing underlying high-grade cervical pathology among older women if left unscreened.

Cervical cytology remains the principal screening method to prevent cervical cancer. In spite of the known limitations, a significant reduction in mortality to cervical cancer has been demonstrated in countries where well established cytology-based screening program has been in place (Bosch and Harper 2006). Colposcopy is one of the most important methods for managing women with abnormal cervical cytology. Immediate colposcopy after abnormal cytology allows underlying significant lesions to be detected and treated in a timely fashion (Manopunya, et al., 2010). This major advantage is especially beneficial for area with a high incidence of cervical cancer such as Thailand because the underlying invasive lesions are notably high across all grades of cervical smear abnormalities (Kietpeerakool et al., 2008; Kietpeerakool, et al., 2014).

As clearance rate of HR-HPV infection decreased with increasing age (Bosch and Harper 2006; Kjaer, et al., 2010; Kang, et al., 2014), we hypothesized that older women with abnormal cervical cytology has an increased risk of harboring underlying significant pathology. This study was conducted to determine whether advancing age is an independent predictor of increased risk of high-grade pathology results among women referred for colposcopy after abnormal cervical cytology.

\section{Materials and Methods}

In this retrospective study, medical records were 


\section{Chalita Kingnate et al}

reviewed for women with abnormal cervical screening results who underwent colposcopy at the Colposcopy Clinic, Srinagarind Hospital, Faculty of Medicine, Khon Kaen University, Thailand, between January and December, 2011. The study was approved by the Research Ethics Committee of the Faculty. Because it was a retrospective study and the data were analyzed anonymously, the need for informed consent was waived by the Research Ethics Committee.

Women were excluded if they were pregnant, had a previous history of abnormal cervical cytology, or underwent colposcopy for other indications (e.g. follow-up after treatment or postcoital bleeding). Data included baseline characteristics, types of cervical smear abnormality, colposcopic findings, subsequent management and detailed histopathologic results.

During the study period, it is our policy to perform colposcopy in all women with abnormal cervical cytology regardless of severity of smear abnormality. Colposcopic examination was performed following the application of $5 \%$ acetic acid solution on the upper vagina and cervix. The severity of colposcopic findings was based on the density of acetowhite areas, sharpness of the lesion margins, and abnormal and atypical vascular patterns. Traditionally, a colposcopically-directed biopsy (CDB) was taken from the area of greatest abnormal appearance, and endocervical curettage (ECC) was carried out if endocervical lesion was suspected. Cervical conization was performed if the initial work-up results were positive for high-grade lesions. Alternatively, loop electrosurgical procedure was immediately carried out if either preceding cervical smears or colposcopic impressions revealed highgrade abnormality.

The final histological diagnosis was made on the most severe histological results obtained after initial colposcopy. Women with normal colposcopic findings without CDB or ECC were categorized as having no significant lesion. High-grade pathology results included cervical intraepithelial neoplasia (CIN) 2-3, adenocarcinoma in situ (AIS), endometrial hyperplasia, and cancer of any original site. For each patient, surgical specimens were examined by the gynecologic pathologists (P.K.). Women with invasive cancer were staged according to the International Federation of Gynecology and Obstetrics (FIGO) classification .

Statistical analysis was carried out with SPSS software (IBM, Armonk, NY, USA). Descriptive statistics were used for demographic baseline characteristics. The $\chi^{2}$ and Fisher's exact test were calculated whenever appropriate to compare the groups. Logistic regression was used as a multivariate analysis to determine an independent impact of patients' age on the risk of encountering highgrade pathology results. Collinearity between the factors included in the multivariate analyses was checked. An odds ratio with a $95 \%$ confidence interval (CI), which did not include unity were considered statistically significant.

\section{Results}

During the study period, 482 consecutively women who met the inclusion criteria were reviewed. The mean age of the women was 42.8 years (range, 18-73 years). Of 482 women, 97 (20.1\%) were postmenopausal, and 92 $(19.1 \%)$ were nulliparous.

Table 1 shows the baseline characteristics of the study patients. The most common type of cervical smear abnormality was atypical squamous cell of undetermined significance (ASC-US) $(\mathrm{n}=170)$, followed by low-grade squamous intraepithelial lesion (LSIL) $(n=128)$, and highgrade squamous intraepithelial lesion (HSIL) $(n=102)$. Of 482 women, high-grade pathology results included CIN 2-3, $99(20.5 \%)$, AIS , $4(0.8 \%)$, cervical cancer, $30(6.2 \%)$, and endometrial cancer, $1(0.2 \%)$. The prevalence of high-grade pathology results was $26.9 \%$ (95\% CI, $23.1 \%$ $31.2 \%)$. In total, 31 women were found to have cancers in histologic follow-up $(6.4 \%$; 95\% CI, $4.4 \%-9.0 \%)$. The FIGO stage of these 30 cases who found to have cervical cancer included stage IA1 (15), IA2 (1), IB 1 (9), IIA1 (1), and IIB (4). One woman who had grade I endometrioid adenocarcinoma of the endometrium was in stage IB.

Table 2 displays the type of smear abnormality stratified by patients' age and parity status. High-grade squamous cell abnormality on cervical smears was more common among older than younger women. Similarly, abnormal cervical smears suggesting glandular lesion was more commonly among older than younger women. The

\section{Table 1. Baseline Characteristics of the Patients} $(n=482)$

\begin{tabular}{lr}
\hline Characteristics & Number (percentage) \\
\hline Parity status & $390(80.9)$ \\
Multiparous & $92(19.1)$ \\
Nulliparous & \\
Menopausal status & $97(20.1)$ \\
Postmenopause & $385(79.9)$ \\
Premenopause & \\
Smear type & $170(35.3)$ \\
ASC-US & $24(5.0)$ \\
ASC-H & $128(26.6)$ \\
LSIL & $102(21.2)$ \\
HSIL & $11(2.3)$ \\
Squamous cell carcinoma & $43(8.9)$ \\
AGC & $1(0.2)$ \\
AIS & $3(0.6)$ \\
Adenocarcinoma & \\
Procedure & $57(11.8)$ \\
Colposcopy only & $276(57.3)$ \\
CDB \pm ECC & $106(22.0)$ \\
Conization \pm ECC & $40(8.3)$ \\
ECC & $3(0.6)$ \\
ECC with endometrial sampling & \\
Final histopathology & $206(42.7)$ \\
No lesion & $142(29.5)$ \\
CIN 1 & $99(20.5)$ \\
CIN 2-3 & $4(0.8)$ \\
AIS & $30(6.2)$ \\
Cervical cancer & $1(0.2)$ \\
Endometrial cancer & \\
\hline ASC-US, atypicas sqamour & \\
\hline
\end{tabular}

ASC-US, atypical squamous cell of undetermined significance; ASC-H, atypical squamous cell cannot exclude high-grade squamous intraepithelial lesion; LSIL, low-grade squamous intraepithelial lesion; HSIL, high-grade squamous intraepithelial lesion; AIS, adenocarcinoma in situ; AGC, atypical glandular cell; CDB, colposcopically-directed biopsy; ECC, endocervical curettage; CIN, cervical intraepithelial neoplasia 
cytology suggesting high-grade squamous and glandular cells abnormality were more common among multiparous than nulliparous women.

Table 2. Type of smear abnormality cross-tabulated with age group and parity status

\begin{tabular}{|c|c|c|c|}
\hline \multirow[t]{2}{*}{ Variables } & \multicolumn{3}{|c|}{ Severity of smear abnormality } \\
\hline & $\begin{array}{l}\text { LGSL }^{a} \\
(n=298)\end{array}$ & $\begin{array}{l}\text { HGSL }^{b} \\
(n=137)\end{array}$ & $\begin{array}{c}\text { Glandular } \\
\text { lesion }{ }^{\mathrm{c}}(\mathrm{n}=47)\end{array}$ \\
\hline \multicolumn{4}{|l|}{ Age (years) } \\
\hline $1<35$ & $75(25.1)$ & $28(20.4)$ & $4(8.5)$ \\
\hline$\geq 35$ & $223(74.8)$ & $109(79.5)$ & $43(91.5)$ \\
\hline $2<40$ & $116(38.9)$ & $48(35.0)$ & 7 (14.9) \\
\hline$\geq 40$ & $182(61.1)$ & $89(65.0)$ & $40(85.1)$ \\
\hline \multicolumn{4}{|l|}{ Parity status } \\
\hline Multiparous & $232(77.8)$ & 117 (85.4) & $41(87.2)$ \\
\hline Nulliparous & $66(22.2)$ & $20(14.6)$ & $6(12.8)$ \\
\hline
\end{tabular}

Table 3. Distribution of Cervical Histopathology Results Stratified by Age Group, Smear Type, and Parity Status

\begin{tabular}{lrrr}
\hline Variables & \multicolumn{3}{c}{ Histopathology results } \\
\cline { 2 - 4 } & $\begin{array}{c}\text { No lesion/CIN1 } \\
(\mathrm{n}=348)\end{array}$ & $\begin{array}{c}\text { CIN 2-3/AIS } \\
(\mathrm{n}=103)\end{array}$ & $\begin{array}{r}\text { Cancer } \\
(\mathrm{n}=31)\end{array}$ \\
\hline Age (years) & $79(22.7)$ & $27(26.2)$ & $1(3.2)$ \\
$1<35$ & $269(77.3)$ & $76(73.8)$ & $30(96.7)$ \\
$\geq 35$ & $122(35.1)$ & $43(41.7)$ & $6(19.4)$ \\
$2<40$ & $226(64.9)$ & $60(58.3)$ & $25(80.6)$ \\
$\geq 40$ & & & \\
Parity status & $272(78.2)$ & $88(85.5)$ & $30(96.7)$ \\
$\quad$ Multiparous & $76(21.8)$ & $15(16.3)$ & $1(3.2)$ \\
$\quad$ Nulliparous & & & \\
Smear abnormality & $274(78.7)$ & $24(23.3)$ & $0(0)$ \\
$\quad$ LGSL & $42(12.1)$ & $74(71.8)$ & $21(67.7)$ \\
HGSL & $32(9.2)$ & $5(4.9)$ & $10(32.3)$ \\
$\quad$ Glandular lesionc & 32 &
\end{tabular}

CIN, cervical intraepithelial neoplasia; AIS, adenocarcinoma in situ; LGSIL, low-grade squamous intraepithelial lesion; HGSIL, high-grade squamous intraepithelial lesion; ${ }^{\text {a }}$ LGSL, low-grade squamous lesion including ASC-US (170) and LSIL (128); ${ }^{\mathrm{b}} \mathrm{HGSL}$ including ASC-H (24), HSIL (102), and squamous cell carcinoma (11); ${ }^{\mathrm{c}}$ Including AGC (43), AIS (1), and adenocarcinoma (3)
Table 3 shows the prevalence of underlying high-grade histopathology results in different groups of the study patients. Prevalence of high-grade pathology results (CIN 2-3, AIS, and cancer) among women with cervical smears suggesting high-grade squamous cell and glandular cell abnormality was significantly higher than those who had low-grade squamous cell abnormality smears $(59.8 \%$ versus $8.1 \%, \mathrm{P}<0.01)$. These significant histopathology results were found more commonly in multiparous women ( $30.3 \%$ versus $17.4 \%, \mathrm{P}=0.01$ ). Prevalence of significant pathology results among postmenopausal women was $32.0 \%$ which did not significantly differ from that of premenopausal women $(26.8 \%, \mathrm{P}=0.31)$. When adjusted for severity of cervical smear abnormality and parity status, age was noted to be a significant independent predictor of high-grade histopathology. Women older than 35-40 years were approximately 2 times as likely to have underlying severe histopathology as those younger women (Table 4).

\section{Discussion}

One important issue of managing women with abnormal cervical smears is to identify patients at greatest risk of harboring high-grade lesions. In the present study, the rate of underlying significant histopathology after colposcopic evaluation among women with abnormal cervical smears and the associated risk factors have been systematically evaluated. The rate of significant histopathology in this study was notably high $(27.7 \%)$, particularly for invasive cancer (6.4\%). Patients' age was a significant independent factor for predicting the risk of harboring high-grade histopathology results when controlling for applied prognostic factors.

Previous studies have attempted to determine the impact of patients' age on the risk of underlying highgrade pathologies. However, existing data are unevenly contradictory (Sawaya et al., 2000; Louro, et al., 2003; Barreth et al., 2006; Bhogireddy et al., 2014). Findings reported from the populations residing in the areas with adequate coverage rate of cervical cancer screening suggest that older women with abnormal cervical cytology trend to have a lower rate of underlying significant pathologies than younger women (Sawaya, et al., 2000; Louro, et al., 2003; Barreth, et al., 2006). Sawaya et al. (2000) reported

Table 4. Impact of Patients' Age on the Risk of Encountering High-Grade Pathology Results Adjusted by Severity of Smear Results And Parity Status

\begin{tabular}{|c|c|c|c|c|c|}
\hline & \multirow{4}{*}{ Age group } & & & \multirow{2}{*}{\multicolumn{2}{|c|}{ Multivariate analyses }} \\
\hline & & \multicolumn{2}{|c|}{ histopathology results } & & \\
\hline & & CIN 2-3/AIS & Cancer & Adjusted OR & \multirow{2}{*}{ P-value } \\
\hline & & $(n=103)$ & $(n=31)$ & $(95 \% \mathrm{CI})$ & \\
\hline \multicolumn{6}{|c|}{ Cut-off (years) } \\
\hline \multirow{2}{*}{1} & $\geq 35$ & 76 & 30 & $2.04(1.02-4.07)$ & \multirow{2}{*}{0.04} \\
\hline & $<35$ & 27 & 1 & Reference & \\
\hline \multirow{2}{*}{2} & $\geq 40$ & 60 & 25 & $1.99(1.14-3.47)$ & \multirow{2}{*}{0.02} \\
\hline & $<40$ & 43 & 6 & Reference & \\
\hline
\end{tabular}

*CIN, cervical intraepithelial neoplasia; AIS, adenocarcinoma in situ; OR, odds ratio; CI, confidence interval 
that the majority of older US women recruited in the Heart and Estrogen/progestin Replacement Study (HERS) with abnormal cervical smears were found to have negative or low-grade lesion on histologic evaluation. Previous studies conducted among US women noted a significantly higher detection rate of biopsy-proven high-grade lesion following a cytologic interpretation of ASC-H smears in women younger than 35-40 years (Louro, et al., 2003; Barreth, et al., 2006).

Paradoxically in this study which conducted in area having a high incidence of cervical cancer (Moore et al., 2010), our data demonstrate a notably high prevalence of significant lesion among women with abnormal cervical cytology within an age cohort reported by some to be a lower risk. A remarkably high proportion of underlying high-grade pathologies were diagnosed among women older than 35-40 years across all grades of cervical smear abnormalities. In addition, almost all of women who had invasive lesion were 40 years or older. We also were able to demonstrate a significant independent effect of patients' age for predicting the risk of harboring high-grade histopathology results after controlling for type of smear abnormality and parity. Women older than 35-40 years were approximately 2 times as likely to have underlying severe histopathology as those younger women. Like us, findings of the recent study conducted in the underserved community setting reported that CIN 2-3 and cancer were more frequently observed among women aged 35 years or older (Bhogireddy et al., 2014). The authors postulated that a higher risk of encountering high-grade pathologies among older women may be due to the fact that these underserved women were less likely to accomplish with regular screening thus allowing such clinically significant lesions to develop and progress (Bhogireddy et al., 2014). These findings suggest some important practical considerations about the need of an aggressive management to ensure exclusion of underlying high-grade pathologies, particularly invasive cancer among older women coming from underserved or low screening coverage settings.

In this study, the rate of significant histopathology results were also found more commonly in multiparous women (30.3\% versus $17.4 \%, \mathrm{P}=0.01)$. Similarly, Kiatiyosnusorn et al. (2010) noted that parity status was an independent predictor for high-grade disease on initial colposcopic evaluation in women with LSIL smears. Compared to nulliparous women, mutiparous women had 2.6 times the risk of CIN 2-3 or worse. In previous epidemiologic study evaluating the impact of reproductive factors on the risk of cervical cancer, exposure to continuously high level of estrogen has been proposed as a potential factor promoting transformation from HRHPV infection to neoplastic epitheliums (Vaccarella et al., 2006). More frequently exposure to an elevated estrogen levels during pregnancy therefore may be underlying mechanism of an increased risk of developing high-grade cervical disease among multiparous women.

Previous studies demonstrated a relatively high prevalence of underlying significant pathology, particularly invasive cancer among Thai women with abnormal cervical smears even in those having only minimal smear abnormality (Kietpeerakool et al., 2008; Aue-Aungkul et al., 2011; Kietpeerakool et al., 2014; Paengchit et al., 2014). Similarly, interesting finding in our study was the high prevalence of significant lesions $(27.7 \%)$, particularly invasive cancer $(6.4 \%)$. This may reflect the impact of individual background incidence of cervical cancer on the cytopathologic correlation. This baseline information is crucial and must be taken into consideration in management of women with abnormal cytological screening.

Some limitations of this study are worthy of note. This study was retrospective; some clinically important data therefore may not be available, such as contraceptive practice, smoking habit, detailed sexual behavior, and history of previous screening which might influence to the rate of underlying lesions. A lack of HR-HPV testing is another limitation. Results from this study could, however, be generalized in our actual situation and probably in other low-resource areas since HPV testing may not be routinely available.

In conclusion, our study illustrates the substantial risk of underlying significant lesions especially invasive cancer in Thai women with abnormal cervical cytology. Patients' age was a significant independent factor predicting the risk of harboring high-grade histopathology results after controlling for type of cervical smear abnormality and parity status.

\section{Acknowledgements}

We would like to acknowledge Prof. James A Will for editing the MS via Publication Clinic, Khon Kaen University.

\section{References}

Aue-Aungkul A, Punyawatanasin S, Natprathan A, et al (2011). See and treat approach is appropriate in women with highgrade lesions on either cervical cytology or colposcopy. Asian Pac J Cancer Prev, 12, 1723-6.

Barreth D, Schepansky A, Capstick V, et al (2006). Atypical squamous cells-cannot exclude high-grade squamous intraepithelial lesion (ASC-H): a result not to be ignored. $J$ Obstet Gynaecol Can, 28, 1095-8.

Bhogireddy V, Roston A, Chor J, et al (2014). Cervical intraepithelial neoplasia and cancer in women 35 years and older. J Low Genit Tract Dis, 18, 41-5.

Bosch X, Harper D (2006). Prevention strategies of cervical cancer in the HPV vaccine era. Gynecol Oncol, 103, 21-4.

Chen HC, Schiffman M, Lin CY, et al (2011). Persistence of type-specific human papillomavirus infection and increased long-term risk of cervical cancer. J Natl Cancer Inst, 103, 1387-96.

Dalstein V, Riethmuller D, Pretet JL, et al (2003). Persistence and load of high-risk HPV are predictors for development of high-grade cervical lesions: a longitudinal French cohort study. Int J Cancer, 106, 396-403.

Kang LN, Castle PE, Zhao FH, et al (2014). A prospective study of age trends of high-risk human papillomavirus infection in rural China. BMC Infect Dis, 14, 96.

Kiatiyosnusorn R, Suprasert P, Srisomboon J, et al (2010). Highgrade histologic lesions in women with low-grade squamous intraepithelial lesion cytology from a region of Thailand with a high incidence of cervical cancer. Int J Gynaecol 
Obstet, 110, 133-6.

Kietpeerakool C, Srisomboon J, Tantipalakorn C, et al (2008). Underlying pathology of women with "atypical squamous cells, cannot exclude high-grade squamous intraepithelial lesion" smears, in a region with a high incidence of cervical cancer. J Obstet Gynaecol Res, 34, 204-9.

Kietpeerakool C, Tangjitgamol S, Srisomboon J (2014). Histopathological outcomes of women with abnormal cervical cytology: a review of literature in Thailand. Asian Pac J Cancer Prev, 15, 6489-94.

Kjaer SK, Frederiksen K, Munk C, et al (2010). Long-term absolute risk of cervical intraepithelial neoplasia grade 3 or worse following human papillomavirus infection: role of persistence. J Natl Cancer Inst, 102, 1478-88.

Louro AP, Roberson J, Eltoum I, et al (2003). Atypical squamous cells, cannot exclude high-grade squamous intraepithelial lesion. A follow-up study of conventional and liquid-based preparations in a high-risk population. Am J Clin Pathol, 120, 392-7.

Manopunya M, Suprasert P, Srisomboon J, et al (2010). Colposcopy audit for improving quality of service in areas with a high incidence of cervical cancer. Int J Gynaecol Obstet, 108, 4-6.

Moore MA, Attasara P, Khuhaprema T, et al (2010). Cancer epidemiology in mainland South-East Asia - past, present and future. Asian Pac J Cancer Prev, 11, 67-80.

Paengchit K, Kietpeerakool C, Lalitwongsa S (2014). Prevalence and genotype distribution of HPV among women attending a cervical canceer screening mobile unit in Lampang, Thailand. Asian Pac J Cancer Prev, 15, 6151-4.

Rodriguez AC, Garcia-Pineres AJ, Hildesheim A, et al (2011). Alterations of T-cell surface markers in older women with persistent human papillomavirus infection. Int $J$ Cancer, 128, 597-607.

Sawaya GF, Grady D, Kerlikowske K, et al (2000). The positive predictive value of cervical smears in previously screened postmenopausal women: the Heart and Estrogen/progestin Replacement Study (HERS). Ann Intern Med, 133, 942-50.

Vaccarella S, Herrero R, Dai M, et al (2006). Reproductive factors, oral contraceptive use, and human papillomavirus infection: pooled analysis of the IARC HPV prevalence surveys. Cancer Epidemiol Biomarkers Prev, 15, 2148-53. 